\title{
A Mathematical Model and Heuristic Approach for the Production Planning in the Glass Container Industry
}

\author{
Flaviana Moreira de Souza Amorim ${ }^{1}$ \\ ICMC, University of São Paulo - USP, Brazil \\ Márcio da Silva Arantes ${ }^{2}$ \\ ICMC, University of São Paulo - USP, Brazil \\ Claudio Fabiano Motta Toledo ${ }^{3}$ \\ ICMC, University of São Paulo - USP, Brazil \\ Bernardo Almada-Lobo ${ }^{4}$ \\ INESC, University of Porto - UP, Portugal
}

\begin{abstract}
This work presents a mathematical model for production planning problem in the glass containers industry. The manufacturing process in this type of industrial plant has two phases. In the first phase, the glass melting in a furnace is performed and in the second stage the production of containers occurs in molding machines. The appropriate configurations for each machine connected in a furnace is a decision to be taken, which depends on the demand forecast for glass container within a time horizon. The model proposed will describe objectives and constraints that allow defining the best configuration for the whole plant. A heuristic combining Fix-and-Optimize and Relax-and-Fix approaches is also applied over the same instances. The results indicate that the proposed model as well as the heuristics are able to provide good quality solutions for this problem.
\end{abstract}

keywords. Glass Container Industry, Mathematical Modeling, Production Planning, Heuristics, Relax-and-Fix, Fix-and-Optimize.

\section{Introduction}

The problem studied on this paper deals with how to determine a better configuration for an industrial plant that produces glass containers. The lot-sizing decisions must satisfy constraints related with machines and furnace capacities. Currently, production planning can reflect the possible specificity of industrial processes and allow greater flexibility for production operations [10].

The main contribution of this work is to propose a mathematical formulation, based on mixed integer programming model, capable of describing the production situation studied on this paper. A set of instances is generated from data provided by a glass container

\footnotetext{
1 joflaviana@yahoo.com.br

${ }^{2}$ marcio.da.silva.arantes@gmail.com

${ }^{3}$ claudio@icmc.usp.br

${ }^{4}$ almada.lobo@fe.up.pt
} 
industry located in Portugal. The heuristics is based on mathematical programming and combines the Relax-and-Fix (R\&F) along with Fix-and-Optimize (F\&O) approaches.

\section{Related Work}

The glass container problem considered in this paper includes lot-sizing decisions. There are several reviews in the literature about lot sizing and production planning as [6] and [12]. A review about models for lot sizing problems is presented in [10], while another review considering meta-heuristics applied to lot sizing problems can be found at [11]. In the context of lot-sizing problem in the glass container industry, the authors in [1] treat a system with multiple production facilities where a mathematical model is presented. In [2] and [15], it is described a mathematical formulation and meta-heuristic methods for the short-term production planning in a single-facility with only one furnace.

The present paper applies a heuristics based on mathematical programming that combines the construction heuristics relax-and-fix (R\&F) with the improvement heuristics fix-and-optimize ( $\mathrm{F} \& \mathrm{O})$. A review on mathematical programming based heuristics is presented in [4]. The construction method R\&F solves mixed-integer programming (MIP) sub models, optimizing some integer variables and keeping others relaxed [17]. The authors in [3] applies R\&F heuristics to solve a lot sizing problem in the foundry industry. A similar approach is applied by [16] and [5] for a lot sizing problem in the animal feed industry. In [7] and [8], R\&F is also used to solve a production problem in the beverage industry. The improvement $\mathrm{F} \& \mathrm{O}$ heuristics starts from an initial solution, wheres some of its variables are re-optimized while others are fixed [17]. In [14], F\&O was combined with an genetic algorithm to solve also a multi-stage lot sizing problem.

\section{Mathematical model}

This section presents the mathematical model, where it is assumed only one furnace that can be connected to several machines with different configurations. The parameters and decision variables are shown next.

\section{Parameters:}

- $m$ : Machines available $(m=1, \ldots, M)$.

- $i$ : Products to be manufactured $(i=1, \ldots, I)$.

- $a$ : Annual Time horizon $(a=1, \ldots, A)$.

- $N S_{m}$ : Number of sections by machine $m$.

- $T G_{m}$ : Type of gob by machine $m$.

- $A C_{i m}: 1$ if product $i$ is accepted in the machine $m$.

- $C_{m}$ : Cost to install machine $m$. $(\$$ 
- $D_{i a}$ : Demand expected of product $i$ in period $a$. (ton)

- $W_{i}$ : Weight of product $i$. (ton)

- $R_{i}$ : Efficiency of the cavity for product $i$. (bottles/min)

- $\bar{M}$ : Maximum machines supported by the new furnace.

- $C F$ : Cost to install fuse capacity on furnace. (\$/ton)

- $\eta_{m}$ : Efficiency of machine $m$. (\%)

\section{Variables:}

- $K F$ : Melting capacity required for the furnace. (ton)

- $Q_{i m a}$ : Lot size of product $i$ on machine $m$ in the period $a$. (ton)

- $F_{\text {ima }}$ : Time spent on period $a$ in which machine $m$ was dedicated to produce product $i$. (years)

- $\bar{Y}_{m}: 1$ if the machine $m$ is installed, 0 otherwise.

\section{Formulation:}

$$
\text { Min } \quad f\left(K F, \bar{Y}_{1}, \ldots, \bar{Y}_{M}\right)=C F * K F+\sum_{m=1}^{M} C_{m} . \bar{Y}_{m}
$$

Subject to:

$$
\begin{array}{lr}
\sum_{m=1}^{M} \bar{Y}_{m} \leq \bar{M} & \\
F_{\text {ima }} \leq \bar{Y}_{m} & \forall(i, m, a) \\
F_{\text {ima }} \leq A C_{i m} & \forall(i, m, a) \\
\sum_{i} F_{i m a}=\bar{Y}_{m} & \forall(m, a) \\
Q_{i m a}=F_{i m a} \cdot\left(R_{i} \cdot W_{i} \cdot N S_{m} \cdot T G_{m} \cdot \eta_{m}\right) & \forall(i, m, a) \\
\sum_{\tau=1}^{a} \sum_{m} Q_{i m \tau} \geq \sum_{\tau=1}^{a} D_{i \tau} & \forall(i, a) \\
\sum_{i} \sum_{m} Q_{i m a} \leq K F & \forall(i, a) \\
K F, Q_{i m a}, F_{i m a} \geq 0 & \\
\bar{Y}_{m} \in\{0,1\} &
\end{array}
$$

The objective function (1) minimizes costs related to increase the melting capacity of the furnace along with the cost of machines. Constraints (2) represent the limit of machines that can be installed in the furnace. Constraints (3) allow the production of product $i$ only if machine $m$ is installed, while constraints (4) allow the production only if the product $i$ is accepted by machine $m$. The setup time between products for each period $a$ and machine $m$ is described by constraints (5). The lot-size of product $i$ in machine $m$ in period $a$ is defined in constraints (6), while constraints (7) ensure the demand satisfaction. Constraints (8) define that the furnace and machine capacities must meet the demand. The variable domains are given by constraints (9) and (10). 


\section{Methods}

Algorithm 1 describes the R\&F developed, where a partition $(\hat{m})$ defines the set of binary variable to be optimized while the others remain relaxed. At the end of each iteration, the binary variables have their values fixed and another partition is defined.

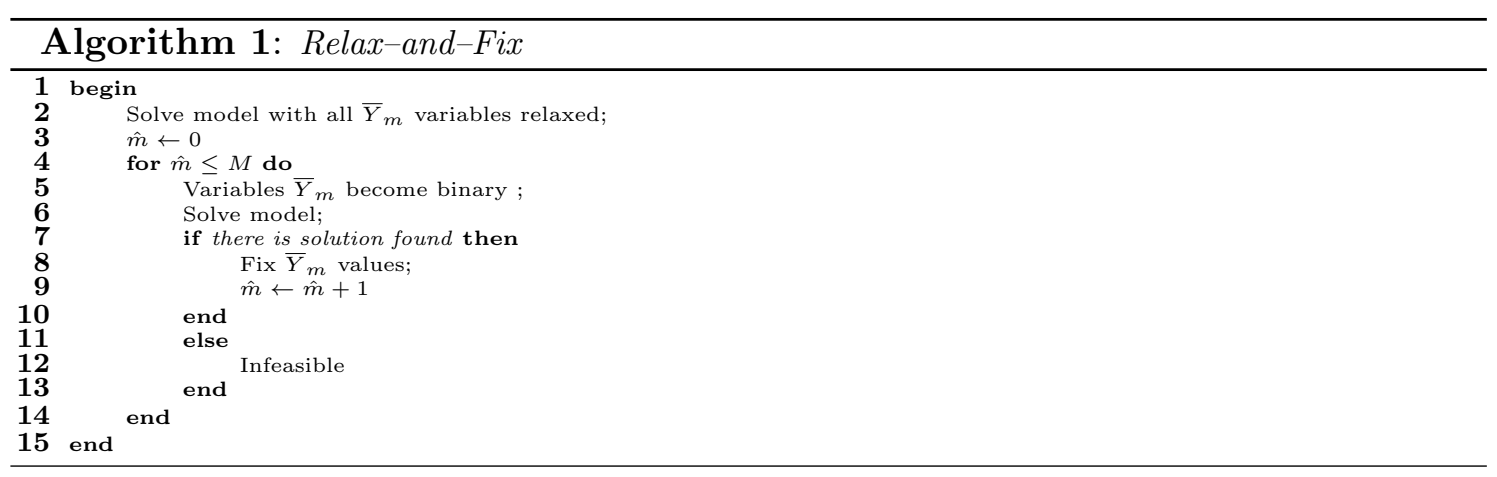

Algorithm 2 describes the $\mathrm{F} \& \mathrm{O}$, where the final solution of $\mathrm{R} \& \mathrm{~F}$ is used as initial solution. Of course, if $\mathrm{R} \& \mathrm{~F}$ did not find a feasible solution, $\mathrm{F} \& \mathrm{O}$ will be also infeasible once it is a improvement heuristic and needs an initial solution. A partition $(\hat{m})$ defines the set of binary variable to be optimized while the others remain relaxed. At the end of each iteration, the binary variables have their values fixed and another partition is defined.

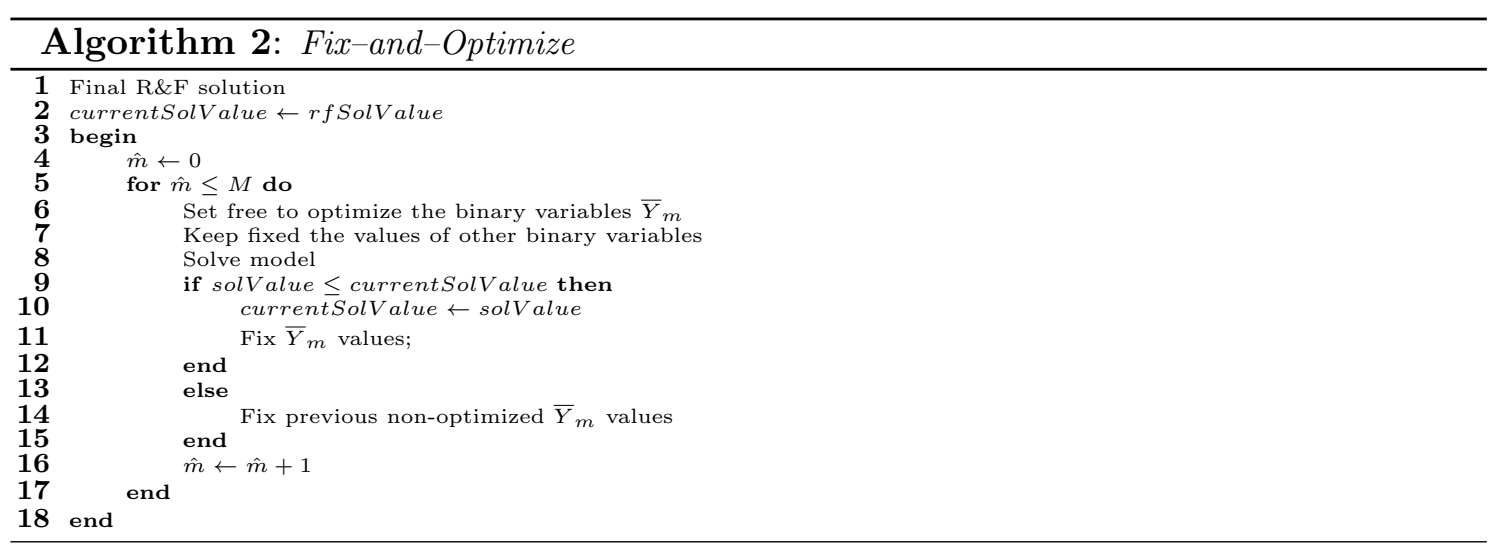

\section{Computational Results}

The instances to be evaluated were created by a random generator from data provided by a glass container industry located in Portugal. Two groups of instances were elaborated: Factory-Machine (FM) and Horizon-Time (HT). A total of five subgroups of instances were created for each case, where each subgroup contains 10 instances.

The mixed integer model proposed was coded in $\mathrm{C} / \mathrm{C}++$ using the interface OPL of package IBM ILOG CPLEX 12.6. The interface OPL and solver CPLEX were also used 
to develop the heuristics $\mathrm{R} \& \mathrm{~F}$ with $\mathrm{F} \& \mathrm{O}$. The exact solution from model formulation and the solutions returned by $\mathrm{R} \& \mathrm{~F}$ with $\mathrm{F} \& \mathrm{O}$ were tested in a total of 100 instances, where 50 instances come from FM and 50 from HT. The time limit was one hour to run each method. All tests were performed on a computer with an Intel(R) Core(TM) i7, 2,67 GHz e 18 GB RAM, and operating system Linux (version 3.2.0-4-amd64 / Debian 4.6.3-14). The resolution of instances from the proposed model, using the solver CPLEX, allowed reaching optimal solutions for some instances as can be observed in Figure 1.

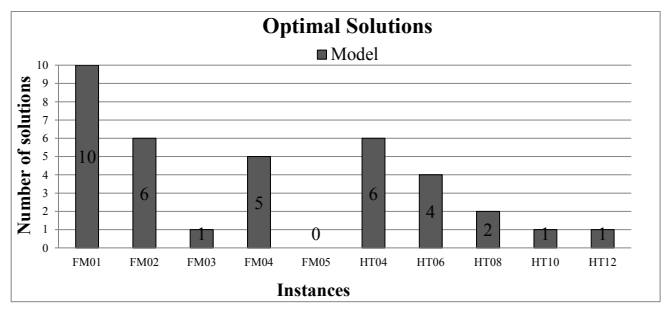

Figure 1: Number of optimal solutions using the proposed model.

In Figure 1, it is observed that solver CPLEX finds 10 optimal solutions in the scenario with 100 machines (FM01). In other scenarios, it was possible to find at maximum 6 optimal solutions. In the HT group, a total of 6 and 4 optimal solutions are obtained for the two simpler scenarios (HT04 and HT06), while we have two or one optimal solution returned within one hour for the other cases. The number of variables and constraints increases for both cases when parameters $M$ and $T$ are increasing. This directly impacts over the exact method performance.

$$
\operatorname{Gap}(\%)=100 * \frac{C P L E X^{U B}-C P L E X^{L B}}{C P L E X^{U B}}
$$
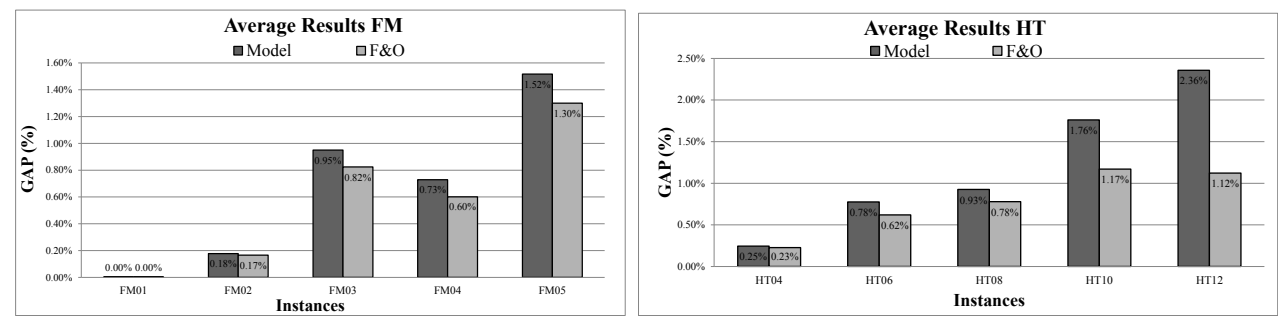

Figure 2: Average results obtained through GAP.

Figure 2 compares the average results obtained through the GAP defined in equation (11). This value is obtained from the difference between the Upper Bound (UB) and Lower Bound (LB), divided by the Upper Bound (LB). For instances of the FM group, the methods were the same for FM01 subgroup, while the R\&F with F\&O heuristics obtained better solutions on average than solver CPLEX for the other subgroups. In the HT instances, the proposed heuristic got better solutions for all subgroups.

Figure 3 presents the number of better solutions returned by some of the methods and the amount of draws. A draw occurs when $\mid$ solMethod $1-\operatorname{solMethod} 2 \mid<0.01$. 


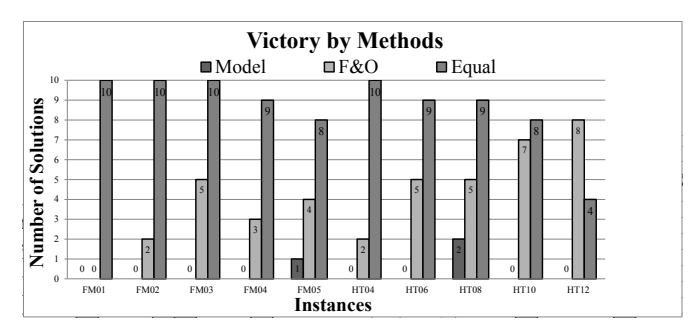

Figure 3: Number of victory and equal between methods.

R\&F with F\&O heuristics wins more than CPLEX for FM group, but several draws also happend. For HT group, there is less number of draws and the propsed heuristic confirms its superiority in terms of number of wins.

\section{Conclusion}

This work introduced a mathematical model where decisions about how to define a plant configuration can be done along with lot size decisions. The mathematical model was able to solve optimally only subgroup FM01. The proposed heuristics is promising once its average GAPs are better for 9 out of 10 sub-groups. There are several draws among the final solutions returned by using the mathematical formulation introduced and the proposed heuristics. However, R\&F with F\&O heuristics returned the best solutions when there are no draws.

\section{Acknowledgment}

The authors would like to thank CAPES, CNPq (grant 483474/2013-4) for supporting the development of this work. The last author would like to thank the ERDF - European Region Development Fund, through the COMPETE program and national funds by FCT - Foundation for Science and Technology, under the framework of the project NORTH-070124-FEDER-000057.

\section{References}

[1] B. Almada-Lobo, J. Oliveira and M. Carravilla, Production planning and scheduling in the glass container industry: A VNS approach, International Journal of Production Economics 114 (1) 363-375, 2008.

[2] B. Almada-Lobo, D. Klabjan, M.A. Carravilla, and J.F. Oliveira, Multiple machine continuous setup lotsizing with sequence dependent setups, Computational Optimization and Applications, 47(3):529-552, 2010.

[3] S. Araujo, M. Arenales and A. Clark, Lot Sizing and Furnace Scheduling in Small Foundries, Comput. Oper. Res. 35 (3) 916-932, 2008. 
[4] M. Ball, Heuristics based on mathematical programming, Surveys in Operations Research and Management Science 16 (1) 21-38, 2011.

[5] A. Clark, R. Morabito and E. Toso, Production setup-sequencing and lot-sizing at an animal nutrition plant through atsp subtour elimination and patching, Journal of Scheduling 13 (2) 111-121, 2010.

[6] A. Drexl and A. Kimms, Lot-sizing and scheduling - survey and extensions, European Journal of Operational Research, 99:221-235, 1997.

[7] D. Ferreira, R. Morabito and S. Rangel, Solution approaches for the soft drink integrated production lot sizing and scheduling problem, European Journal of Operational Research 196 (2) 697-706, 2009.

[8] D. Ferreira, A. Clark, B. Almada-Lobo and R. Morabito, Single-stage formulations for synchronised two-stage lot sizing and scheduling in soft drink production, International Journal of Production Economics 136 (2) 255-265, 2012.

[9] S. Helber and F. Sahling, A fix-and-optimize approach for the multi-level capacitated lot sizing problem, International Journal of Production Economics, Vol 123, p.247256,2010 .

[10] R. Jans, Solving lot-sizing problems on parallel identical machines using symmetrybreaking constraints, INFORMS Journal on Computing, 21(1):123-136, 2009.

[11] R. Jans and Z. Degraeve, Meta-heuristics for dynamic lot sizing: A review and comparison of solution approaches, European Journal of Operational Research, 177:18551875, 2007.

[12] B. Karimi, S. M. T. F. Ghomi and J. M. Wilson, The capacitated lot sizing problem: a review of models and algorithms, Omega 31 365-378, 2003.

[13] F. Sahling, L. Buschkühl, H. Tempelmeier and S. Helber, Solving a multi-level capacitated lot sizing problem with multi-period setup carry-over via a fix-and-optimize heuristic, Computers \& Operations Research 37 2546-2553, 2009.

[14] C.F.M. Toledo, R.R.R. Oliveira and P.M. FranÃßa, A hybrid multi-population genetic algorithm applied to solve the multi-level capacitated lot sizing problem with backlogging, Computers \& Operations Research 40 (4) 910-919, 2013.

[15] C.F.M. Toledo, M. Arantes, R. Oliveira and B. Almada-Lobo, Glass container production scheduling through hybrid multipopulation based evolutionary algorithm, Applied Soft Computing 13 (3) 1352-1364, 2013.

[16] E. Toso, R. Morabito and A. Clark, Lot Sizing and Sequencing Optimisation at an Animal-feed Plant, Comput. Ind. Eng. 57 (3) 813-821, 2009.

[17] Y. Pochet and L. A. Wolsey, Production Planning by Mixed Integer Programming, Springer Science \& Business Media, New York, 2006. 